\title{
Restriction digest screening facilitates efficient detection of site-directed mutations introduced by CRISPR in C. albicans UME6
}

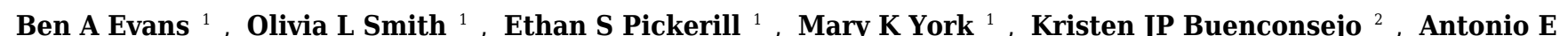
Chambers ${ }^{1}$, Douglas A Bernstein ${ }^{\text {Corresp. } 1}$

1 Department of Biology, Ball State University, Muncie, Indiana, United States

2 Department of Microbiology and Immunology, Drexel University, Philadelphia, Pennsylvania, United States

Corresponding Author: Douglas A Bernstein

Email address: dabernstein@bsu.edu

Introduction of point mutations to a gene of interest is a powerful tool when determining protein function. CRISPR-mediated genome editing allows for more efficient transfer of a desired mutation into a wide range of model organisms. Traditionally, PCR amplification and DNA sequencing is used to determine if isolates contain the intended mutation. However, mutation efficiency is highly variable, potentially making sequencing costly and time consuming. To more efficiently screen for correct transformants, we have identified restriction enzymes sites that encode for two identical amino acids or one or two stop codons. We used CRISPR to introduce these restriction sites directly upstream of the Candida albicans UME6 $\mathrm{Zn}^{2+}$-binding domain, a known regulator of $C$. albicans filamentation. While repair templates coding for different restriction sites were not equally successful at introducing mutations, restriction digest screening enabled us to rapidly identify isolates with the intended mutation in a cost-efficient manner. In addition, mutated isolates have clear defects in filamentation and virulence compared to wild type C. albicans. Our data suggest restriction digestion screening efficiently identifies point mutations introduced by CRISPR and streamlines the process of identifying residues important for a phenotype of interest. 
1 2

3

4

5

6 Ben A. Evans ${ }^{1}$, Olivia L. Smith ${ }^{1}$, Ethan S. Pickerill ${ }^{1}$, Mary K. York ${ }^{1}$, Kristen J.P. Buenconsejo ${ }^{1,2}$, 7 Antonio E. Chambers ${ }^{1}$, Douglas A. Bernstein ${ }^{1}$

8 1. Department of Biology, Ball State University, Muncie, IN, USA

9 2. Department of Microbiology and Immunology, Drexel University, Philadelphia, PA, USA

10 Corresponding Author:

11 Douglas A. Bernstein

12 Email Address: dabernstein@bsu.edu by CRISPR in C. albicans UME6 
30 Introduction of point mutations to a gene of interest is a powerful tool when determining

31 protein function. CRISPR-mediated genome editing allows for more efficient transfer of a desired mutation into a wide range of model organisms. Traditionally, PCR amplification and DNA sequencing is used to determine if isolates contain the intended mutation. However, mutation efficiency is highly variable, potentially making sequencing costly and time consuming. To more efficiently screen for correct transformants, we have identified restriction enzymes sites that encode for two identical amino acids or one or two stop codons. We used CRISPR to introduce these restriction sites directly upstream of the Candida albicans UME6 $\mathrm{Zn}^{2+}$-binding domain, a known regulator of $C$. albicans filamentation. While repair templates coding for different restriction sites were not equally successful at introducing mutations, restriction digest screening enabled us to rapidly identify isolates with the intended mutation in a cost-efficient manner. In addition, mutated isolates have clear defects in filamentation and virulence compared to wild type $C$. albicans. Our data suggest restriction digestion screening efficiently identifies point mutations introduced by CRISPR and streamlines the process of identifying residues important for a phenotype of interest. 
62 Site-directed mutagenesis may be used to introduce a mutation that leads to a change in protein amino acid sequence (Shortle et al. 1981). Investigators can then assess the role of the altered residue/s in particular phenotypes. Site-directed mutagenesis has been used to identify and characterize enzyme active sites, assess the role of amino acid modification on protein function, and characterize a wide variety of additional protein properties (Winter et al. 1982).

Alanine-scanning mutagenesis, where a particular amino acid/s is changed to alanine, is a particularly popular application of this technique (Cunningham \& Wells 1989; Weiss et al. 2000). The methyl sidechain of Alanine is small and nonpolar. The benign nature of the alanine sidechain often abolishes the functionality of the original residue while minimally disrupting protein backbone structure. Additional protein variants can be made in analogous fashions. For instance, in many cases, the carboxyl sidechain of a glutamate residue biochemically mimics a phosphate. Thus, mutation of a threonine or serine to glutamate mimics constitutive phosphorylation (Szewczuk et al. 2009; Thorsness \& Koshland 1987). If an investigator hypothesizes an aromatic or positively charged residue interacts with nucleic acid, a negatively charged or small hydrophobic residue can be introduced to test if said residue is important for nucleic acid binding (Bernstein \& Keck 2005). Moreover, stop codons can be introduced to an open reading frame to generate a $\mathrm{C}$-terminal truncation and a stop codon introduced at the beginning of the open reading frame will terminate translation before a functional protein is made, generating a null protein phenotype. Furthermore, transcription initiation (Vo Ngoc et al. 2017) and splicing (Bortfeldt et al. 2008) are controlled in part by short nucleotide sequences that can be readily interrogated by site-directed mutagenesis. As such, site-directed mutagenesis is a powerful tool to elucidate not only protein but DNA and RNA sequence function.

Historically, site-directed mutagenesis has been a multistep process. In yeast for instance, cells are transformed with exogenous DNA containing a mutation and homologous recombination incorporates the mutant sequence into the genome. Alternatively mutations can be subcloned onto plasmids and transformed into yeast lacking the gene of interest (Cormack \& Castano 2002). These techniques require cells perform homologous recombination at a relatively high rate or maintain plasmids. In addition, detection of single nucleotide polymorphisms (SNPs) between alleles in a diploid is challenging. Incorporation of restriction enzyme cut sites via PCR has been used to detect SNPs using derived cleaved amplified polymorphic sequence analysis (Hodgens et al. 2017; Neff et al. 1998).

One organism that has proven challenging to modify by site-directed mutagenesis is the human fungal pathogen Candida albicans. Because C. albicans is diploid, introduction of a homozygous point mutant has historically required multiple rounds of transformation and homologous recombination (Jones et al. 2004; Muzzey et al. 2013). Furthermore, plasmids are not maintained consistently in C. albicans. The development of the Candida CRISPR system however has made introduction of mutations into the genome more efficient (Vyas et al. 2015; Vyas et al. 2018). During CRISPR-mediated genome editing, Cas9 nuclease bound to a guide RNA molecule targets a specific genomic sequence to be modified using base pairing. Once bound to the complementary sequence, Cas9 introduces a double-strand break. To introduce the desired 
103

mutation and repair the double-strand break by homologous recombination, a double-stranded DNA repair template possessing the altered sequence is cotransformed (reviewed in) (Dominguez et al. 2016; Wang et al. 2016). Clones are sequenced to determine if the desired mutation has been introduced, but efficiency of repair template incorporation can be a substantial bottleneck. Furthermore, DNA sequencing is required to ensure the correct mutation has been introduced into the genome.

We have developed a system to inexpensively screen potential point mutants using restriction digestion. The system enables the investigator to screen for the introduction of two alanine, two glutamate, two arginine, two glycine or one or two stop codons using colony PCR and restriction digestion. As proof of concept, we introduced mutations into UME6, a transcription factor that regulates $C$. albicans filamentation and virulence (Banerjee et al. 2008) and screened for repair template incorporation by PCR and restriction digestion. Specifically, we introduced mutations upstream of sequence encoding the Ume6 $\mathrm{Zn}^{2+}$ finger DNA-binding domain. We found the frequency different repair templates were incorporated into the genome varied significantly. We characterized the effects these mutations had on C. albicans filamentation. Strikingly, introduction of a stop codon directly upstream of the UME6 $\mathrm{Zn}^{2+}$ finger DNA binding domain eliminated filamentation. In addition, we found mutation of two conserved positively charged residues to glutamate directly upstream of the UME6 $\mathrm{Zn}^{2+}$ finger DNA-binding domain resulted in filamentation defects and a decrease in C. albicans virulence.

\section{Materials and Methods:}

Cloning and Sequencing: Primers used in this study are listed in (Table 1). Ume6 guide RNA primers (Ume6 Guide $2 \mathrm{fr}$ and rv) were subcloned into pv1093 as previously described (Vyas et al. 2015). Repair templates were made by PCR using indicated primer pairs. PCR products were purified using a Zymo PCR Purification Kit. Lithium Acetate transformation was performed and cells were plated on yeast extract-peptone-dextrose agar plates that contained $200 \mu \mathrm{g} / \mathrm{ml}$ nourseothricin (Nat) (Gietz \& Woods 2002). All growth media for this study was supplemented with $27 \mathrm{mM}$ uridine. After three days, Nat ${ }^{r}$ colonies were streaked for isolation on YPD medium supplemented with $100 \mu \mathrm{g} / \mathrm{ml}$ Nat. Colony PCR was performed using primers Ume6 ch primer 2 $\mathrm{fr}$ and rv. $10 \mu \mathrm{l}$ of colony PCR product was digested in accordance with the manufacturer's recommended reaction conditions. Digestion and undigested PCR products were resolved on a $2 \%$ agarose gel, stained with ethidium bromide, and visualized. PCR products with digestion patterns consistent with successful mutagenesis were sequenced by GeneWiz, and SNAPgene was used to analyze the sequences.

Growth and Filamentation Assays: C. albicans strains were grown overnight at $25^{\circ} \mathrm{C}$ in YPD and diluted to OD 0.1. Fourfold serial dilutions were plated using a pin replicator on YPD and Spider (Liu et al. 1994). Liquid filamentation was assessed by growing C. albicans variants for 24 hours in Spider Media at $37^{\circ} \mathrm{C}$.

Galleria mellonella Infection: Healthy $(235 \mathrm{mg} \pm 45 \mathrm{mg}$ ) larvae were randomly assigned to sterile PBS, wild type, or mutant groups. Log-phase cultures of C. albicans grown at $37^{\circ} \mathrm{C}$ in YPD were washed twice and suspended in sterile PBS. Prior to each injection, a Hamilton syringe was 
143

144

145

146

147

148

149

150

151

152

153

154

155

156

157

158

159

160

161

162

163

164

165

166

167

168

169

170

171

172

173

sterilized via rinsing once with ethanol and then twice with sterile PBS. Larvae were swabbed with $70 \%$ ethanol. $10 \mu \mathrm{l}$ of either sterile PBS or $10^{5} \mathrm{cfu}$ of $\mathrm{C}$. albicans suspended in sterile PBS was injected per larva into the left rear proleg. Larvae were incubated at $37^{\circ} \mathrm{C}$ in a glass petri dish, and survival was assessed daily by visual inspection and prodding with a sterile pipet tip. Deceased larvae were removed from the dish daily.

\section{Results:}

One of the most challenging and costly aspects of site-directed mutagenesis is screening transformants to identify those harboring the intended mutation. To reduce cost and increase the speed by which transformants can be screened, we identified restriction enzyme recognition sequences that, when introduced, convert two codons to codons encoding two alanines, two arginines, two glutamates, two glycines, stop codons (Table 2). To test our strategy, we cotransformed wild type C.albicans with a plasmid encoding Cas9 and a UME6-targeting guide RNA and PCR-generated repair template DNA with BseRI, Pacl, or Acul recognition sequences. Template-mediated repair replaced UME6 residues 2259-2265 (encoding Lys754 and Lys755) with either two negatively charged glutamate residues (repairEE; BseRI sequence), a TAA stop codon (repairTAA; Pacl sequence), or a TGA stop codon (repairTGA; Acul sequence) (Figure 1, Table 2).

Each transformation resulted in dozens of colonies. We used colony PCR to amplify UME6 from each transformant and digested the PCR products with the enzyme predicted to cut the repair template. 2 of 48 transformants transformed with the repairEE template (designed to replace Lys754/755 with glutamates) were successfully digested by BseRI. 2 of 8 transformants repaired with the repairTAA template (designed to introduce the TAA stop codon) were successfully digested with Pacl, and 2 of 48 transformants repaired with repairTGA (designed to introduce the TGA stop codon) were successfully digested with Acul (Figure 1 B). We sequenced transformants with restriction digestion patterns consistent with successful mutagenesis. We found successfully digested clones had correctly introduced homozygous point mutations in the UME6 gene (Figure $1 \mathrm{D}$ and $\mathrm{E}$ ). In addition, we did not observe unintended mutations in UME6, and we did not recover heterozygous mutants. We also sequenced PCR products that did not digest; as predicted, those transformants retained wild type UME6 sequence (Figure $1 \mathrm{C}$ ). Names and genotypes of strains used in further studies are listed (Table 3). Our data establish that restriction digestion is a rapid and accurate screening strategy for site-directed mutagenesis in C. albicans.

Ume6 is a transcription factor important for $C$. albicans filamentation and the only domain within Ume6 predicted by sequence homology is a $\mathrm{Zn}^{2+}$ finger DNA-binding domain (amino acids 760-810) (Banerjee et al. 2008). Ume6's C-terminal region (amino acids 811-844) is highly conserved, but is not predicted to form a known structural domain (Inglis et al. 2012). We hypothesized the $\mathrm{Zn}^{2+}$ finger and C-terminus of Ume6 are important for filamentation. We found neither yeast harboring the premature stop codon (ume6-stopTAA) nor those expressing the lysine-to-glutamate point mutants (ume6-EE) exhibited slow growth relative to wild type $C$. albicans at a range of temperatures on rich media (Figure 2 B). However, we did find wild type 
183

yeast display a more pronounced crinkled colony morphology than either ume6-EE or ume6stopTAA yeast on rich media at $37^{\circ} \mathrm{C}$ (Figure $2 \mathrm{~B}$ ).

Wild type, but not homozygous ume6/ume6 C. albicans undergo filamentation when cultured on spider medium (Banerjee et al. 2008). We tested if the DNA-binding domain and conserved upstream positively charged residues Lys754 and Lys755 are important for induction of $C$. albicans filamentation. We analyzed filamentation of wild type, ume6-EE, and ume6-stopTAA yeast on spider agar media. We found that, while wild type yeast filament robustly after 7 days, ume6-stopTAA yeast failed to filament (Figure 2 A). Furthermore, we found ume6-EE yeast exhibit intermediate filamentation (Figure $2 \mathrm{~A}$ ). We observed similar filamentation patterns when these strains were cultured in liquid spider media (Figure $2 \mathrm{C}$ ).

Next, we tested the effect of our point mutations on $C$. albicans virulence in a wax moth model. ume6/ume6 yeast exhibit lower virulence in a mouse model (Banerjee et al. 2008), and their virulence has not been tested in the wax moth model system. Less than $30 \%$ of larvae injected with wild type $C$. albicans survived after incubation for 200 hours. By comparison, the ume6stopTAA mutation significantly decreases virulence, with roughly $80 \%$ surviving after 200 hours of incubation (Figure $2 \mathrm{D}$ ). Consistent with our filamentation data, we found ume6-EE yeast exhibited virulence intermediate to wild type and UME6stop-TAA yeast, with greater than 40\% of larvae surviving following 200 hours of incubation. Our data indicate the final 100 amino acids of Ume6 that include both a $\mathrm{Zn}^{2+}$ finger domain and conserved C-terminal region are important for $\mathrm{C}$. albicans filamentation and virulence. Furthermore, our data suggest residues Lys 754 and Lys 755 which lie $\mathrm{N}$-terminal to the $\mathrm{Zn}^{2+}$ finger domain are also important for filamentation and virulence as well.

\section{Discussion:}

We have identified restriction enzyme recognition sequences that, when translated, encode consecutive identical amino acids or one or two stop codons. By replacing two codons with these restriction sites, we were able to efficiently introduce and confirm the change of two consecutive amino acids by PCR followed by restriction digestion. In the event that digestion and sequencing are inconclusive, the incorporation of a restriction site also allows facile screening for correct transformants by Southern Blot, a more time consuming, but potentially necessary step to ensure additional copies of a DNA sequence are not present and your mutation of interest has been introduced. We found not all repair templates are incorporated into the genome with equal efficiency. This variability in successful recombination enhances the utility of rapidly screening numerous colonies by digestion. Our technique enabled us to unambiguously, inexpensively, and rapidly identify desired mutations in the $C$. albicans transcription factor UME6. Although we did not observe heterozygotes, our restriction strategy should distinguish homozygous from heterozygous mutations. Our data suggest that Ume6 Lys754 and Lys755 are important for filamentation and virulence. In addition, our data suggests the $\mathrm{Zn}^{2+}$ finger DNA binding domain and conserved C-terminal domain are also important for filamentation and virulence. 
222 We have identified restriction enzyme recognition sites that encode two consecutive alanines, glutamates, arginines, and glycines and one or two stop codons. Classically, site-directed mutagenesis introduces a single amino acid change, but screening for such changes using restriction digestion is impractical. Introduction of restriction sites with four-base recognition sites (e.g. GCGC which can be cleaved by HinP1l or Hhal) could be used to introduce a single alanine; however, the additional base at the $5^{\prime}$ or $3^{\prime}$ end of the four-base recognition sequence places upstream or downstream sequence constraints that limit its utility.

Introduction of stop codons can be used to truncate the C-terminus of a protein. However, stop codon suppressor mutations could bypass these mutations. The introduction of an I-Scel restriction site introduces two distinct stop codons (TAG and TAA) and is thus less likely to be bypassed with suppressor mutations. We attempted to introduce I-Scel site to UME6 using a 100-base pair repair template and screened over 50 transformants by restriction digestion. Unfortunately, none of these transformants incorporated the repair template. This apparent inefficiency could be due to slightly shortened regions of homology on the repair template in comparison to the successful repair templates. A moderate increase in repair template size might overcome this limitation. Alternatively, successive Spel, Pacl, or Acul restriction sites in a single repair template could be used to introduce sequential stop codons and reduce the likelihood of suppression.

All codons are not used equally in genomes. For instance, in C. albicans GCN (N representing any base) encodes alanine, but $43 \%$ of alanine are encoded by GCT and less than $5 \%$ by GCG (Arnaud et al. 2005). Such codon bias must be appreciated when investigators decide which restriction site to introduce. Rare codons may alter protein expression, confounding the interpretation of phenotypes associated with protein variants (Quax et al. 2015). We have identified three restriction sites that code for successive alanine residues (Table 2). These enzyme recognition sites are found at different frequencies throughout the genome and allow for the introduction of any alanine codon. This cadre of enzymes provides the investigator flexibility when designing a mutagenesis experiment.

Site-directed mutagenesis restriction digest screening could be exceptionally useful for investigation of protein localization. Proteins use short conserved amino acid sequences to localize to a variety of intracellular compartments including the endoplasmic reticulum, mitochondria, peroxisome, and nucleus (Nakai 2000). These sequence motifs are often charged or hydrophobic. Disruption of such sequence motifs is critical when dissecting the function of these proteins in vivo. Site-directed mutagenesis could replace these amino acids with others possessing different biochemical characteristics. Our screening system would enable efficient interrogation of the roles signal motifs play in protein localization.

We piloted our restriction digestion-based strategy to screen for point mutations in UME6. Ume6 is a transcriptional regulator of filamentation, and deletion of UME6 causes defects in filamentation and decreased virulence in mouse models (Banerjee et al. 2008). However, little was known about the function of structural elements within Ume6. Ume6 consists of a predicted $\mathrm{Zn}^{2+}$ finger DNA binding domain, conserved C-terminal domain, and a poorly 
262

263

264

265

266

267

268

269

270

271

272

273

274

275

276

277

278

279

280

281

282

283

284

285

286

287

288

289

290

291

292

293

294

295

296

297

298

299

300

conserved $\mathrm{N}$-terminal domain of unknown function (Figure 1A). Our data show the $\mathrm{Zn}^{2+}$ finger domain and/or the $\mathrm{C}$-terminal domain plays a role in filamentation, as introduction of a stop codon upstream of these domains led to decreased filamentation and virulence (Figure $2 \mathrm{~A}-\mathrm{C}$ ). One potential interpretation is altering conserved lysine residues directly $\mathrm{N}$-terminal to the $\mathrm{Zn}^{2+}$ finger domain causes defects in filamentation and virulence. This suggests Lys754 and/or Lys 755 play a previously unappreciated role in filamentation program activation. Alternatively, changing Lys754 and/or Lys755 could lead to destabilization and/or lower expression of functional protein. The Glu codons that were introduced code for $21 \%$ of Glu residues in Candida so it is unlikely that codon usage is limiting. While Ume6's N-terminal domain is not as highly conserved as its $\mathrm{Zn}^{2+}$ finger or C-terminal domains, it does contain a number of highly conserved residues. Further site-directed mutagenesis of the Ume6 N-terminal domain will help elucidate the role of Ume6 in C. albicans' filamentation program.

Site-directed mutagenesis coupled with restriction digest screening has the potential to be broadly useful in a number of ways. First, the development of CRISPR-mediated genome editing systems has significantly expanded the breadth of organisms that can be readily genetically modified (Reardon 2016). These advances are likely to revolutionize knockout library generation in a variety of organisms. For organisms that require a repair template to introduce mutations like C. albicans (Vyas et al. 2015), introduction of stop codons that can be screened by restriction digestion could be an important component of efficient and accurate library preparation and verification. Second, undergraduate research projects are a critical component of education, but identifying projects that both excite students and can be performed during the confines of an undergraduate curriculum is challenging. This can be especially difficult for students interested in molecular biology, where cloning and reagent development are often times required before one can begin to test a hypothesis experimentally. Introducing mutations using CRISPR and screening for correct mutations using restriction digestion is a quick method whereby undergraduate students can learn and apply multiple molecular biology techniques. The relatively high success rate and moderate cost of these techniques allow students to move projects forward while asking and answering important biological questions. Furthermore, the low cost and speed of these procedures makes these exercises suitable for undergraduate teaching laboratories.

\section{Conclusions:}

We have identified restriction enzymes cut sites that code for successive Alanine, Glutamate, Arginine, Glycine, or stop codons. The incorporation of these sites allows the efficient identification of correct point mutations using restriction digestion. Using CRISPR we introduced these sites to C. albicans UME6, changing Lys754 and Lys755 to either two Glutamate residues or a stop codon. We found Lys754 and Lys755 are important for filamentation and virulence. Site-directed mutagenesis coupled with restriction digest screening is a quick cost effective method of screening potential point mutants.

\section{Acknowledgements}


301 The authors would also like to thank Dr. Gennifer Mager and Dr. Eric (VJ) Rubenstein for helpful 302 comments regarding the manuscript.

References:

Arnaud MB, Costanzo MC, Skrzypek MS, Binkley G, Lane C, Miyasato SR, and Sherlock G. 2005. The Candida Genome Database (CGD), a community resource for Candida albicans gene and protein information. Nucleic Acids Res 33:D358-363. 10.1093/nar/gki003

Banerjee M, Thompson DS, Lazzell A, Carlisle PL, Pierce C, Monteagudo C, LopezRibot JL, and Kadosh D. 2008. UME6, a novel filament-specific regulator of Candida albicans hyphal extension and virulence. Mol Biol Cell 19:1354-1365. 10.1091/mbc.E07-11-1110

Bernstein DA, and Keck JL. 2005. Conferring substrate specificity to DNA helicases: role of the RecQ HRDC domain. Structure 13:1173-1182. 10.1016/j.str.2005.04.018 
332

333

334

335

336

337

338

339

340

341

342

343

344

345

346

347

348

349

350

351

352

353

354

355

356

357

358

359

360

361

362

363

364

365

366

367

368

369
Bortfeldt R, Schindler S, Szafranski K, Schuster S, and Holste D. 2008. Comparative analysis of sequence features involved in the recognition of tandem splice sites. BMC Genomics 9:202. 10.1186/1471-2164-9-202

Cormack B, and Castano I. 2002. Introduction of point mutations into cloned genes. Guide to Yeast Genetics and Molecular and Cell Biology, Pt B 350:199-218. Doi 10.1016/S0076-6879(02)50964-2

Cunningham BC, and Wells JA. 1989. High-Resolution Epitope Mapping of HghReceptor Interactions by Alanine-Scanning Mutagenesis. Science 244:10811085. DOI 10.1126/science. 2471267

Dominguez AA, Lim WA, and Qi LS. 2016. Beyond editing: repurposing CRISPR-Cas9 for precision genome regulation and interrogation. Nat Rev Mol Cell Biol 17:5-15. 10.1038/nrm.2015.2

Gietz RD, and Woods RA. 2002. Transformation of yeast by lithium acetate/singlestranded carrier DNA/polyethylene glycol method. Methods Enzymol 350:87-96.

Hodgens C, Nimchuk ZL, and Kieber JJ. 2017. indCAPS: A tool for designing screening primers for CRISPR/Cas9 mutagenesis events. PLoS One 12:e0188406. 10.1371/journal.pone.0188406

Inglis DO, Arnaud MB, Binkley J, Shah P, Skrzypek MS, Wymore F, Binkley G, Miyasato SR, Simison M, and Sherlock G. 2012. The Candida genome database incorporates multiple Candida species: multispecies search and analysis tools with curated gene and protein information for Candida albicans and Candida glabrata. Nucleic Acids Res 40:D667-674. 10.1093/nar/gkr945

Jones T, Federspiel NA, Chibana H, Dungan J, Kalman S, Magee BB, Newport G, Thorstenson YR, Agabian N, Magee PT, Davis RW, and Scherer S. 2004. The diploid genome sequence of Candida albicans. Proc Natl Acad Sci U S A 101:7329-7334. 10.1073/pnas.0401648101

Liu H, Kohler J, and Fink GR. 1994. Suppression of hyphal formation in Candida albicans by mutation of a STE12 homolog. Science 266:1723-1726.

Muzzey D, Schwartz K, Weissman JS, and Sherlock G. 2013. Assembly of a phased diploid Candida albicans genome facilitates allele-specific measurements and provides a simple model for repeat and indel structure. Genome Biol 14:R97. 10.1186/gb-2013-14-9-r97

Nakai K. 2000. Protein sorting signals and prediction of subcellular localization. Advances in Protein Chemistry, Vol 54 54:277-344. Doi 10.1016/S00653233(00)54009-1

Neff MM, Neff JD, Chory J, and Pepper AE. 1998. dCAPS, a simple technique for the genetic analysis of single nucleotide polymorphisms: experimental applications in Arabidopsis thaliana genetics. Plant J 14:387-392. 
370

371

372

373

374

375

376

377

378

379
Quax TEF, Claassens NJ, Soll D, and van der Oost J. 2015. Codon Bias as a Means to Fine-Tune Gene Expression. Molecular Cell 59:149-161. 10.1016/j.molcel.2015.05.035

Reardon S. 2016. Welcome to the CRISPR zoo. Nature 531:160-163. 10.1038/531160a

Shortle D, DiMaio D, and Nathans D. 1981. Directed mutagenesis. Annu Rev Genet 15:265-294. 10.1146/annurev.ge.15.120181.001405

Szewczuk LM, Tarrant MK, and Cole PA. 2009. Protein Phosphorylation by Semisynthesis: From Paper to Practice. Methods in Enzymology: Non-Natural Amino Acids 462:1-24. 10.1016/S0076-6879(09)62001-2

Thorsness PE, and Koshland DE. 1987. Inactivation of Isocitrate Dehydrogenase by Phosphorylation Is Mediated by the Negative Charge of the Phosphate. Journal of Biological Chemistry 262:10422-10425.

Vo Ngoc L, Wang YL, Kassavetis GA, and Kadonaga JT. 2017. The punctilious RNA polymerase II core promoter. Genes Dev 31:1289-1301. 10.1101/gad.303149.117

Vyas VK, Barrasa MI, and Fink GR. 2015. A CRISPR system permits genetic engineering of essential genes and gene families. Sci Adv 1:e1500248. 10.1126/sciadv. 1500248

Vyas VK, Bushkin GG, Bernstein DA, Getz MA, Sewastianik M, Barrasa MI, Bartel DP, and Fink GR. 2018. New CRISPR Mutagenesis Strategies Reveal Variation in Repair Mechanisms among Fungi. mSphere 3. 10.1128/mSphere.00154-18

Wang H, La Russa M, and Qi LS. 2016. CRISPR/Cas9 in Genome Editing and Beyond. Annu Rev Biochem 85:227-264. 10.1146/annurev-biochem-060815-014607

Weiss GA, Watanabe CK, Zhong A, Goddard A, and Sidhu SS. 2000. Rapid mapping of protein functional epitopes by combinatorial alanine scanning. Proceedings of the National Academy of Sciences of the United States of America 97:8950-8954. DOI 10.1073/pnas.160252097

Winter G, Fersht AR, Wilkinson AJ, Zoller M, and Smith M. 1982. Redesigning enzyme structure by site-directed mutagenesis: tyrosyl tRNA synthetase and ATP binding. Nature 299:756-758. 


\section{Figure 1}

Introduction of restriction sites enable efficient screening for correct point mutants.

A. Cartoon of Ume6 domains and key residues for this study. Red line indicates CRISPR cut site. B. Restriction digestion of colony PCR of UME6 from representative ume6TAAStop and ume6-EE C. albicans. Lane 1. Ladder, 2. ume6TAAStop undigested, 3. ume6TAAStop digested with Pac1 4. ume6-EE undigested 5. ume6-EE digested with BseRI C-E. Sequence analysis of site of ume6 mutagenesis of representative failed mutant (wild type sequence) (C), ume6-EE (D), and ume6TAAStop (E) C. albicans amino acid sequence is presented above DNA sequence. Red E designates site of lysine-to-glutamate point mutations. Red octagon designates site of introduced stop codon. 


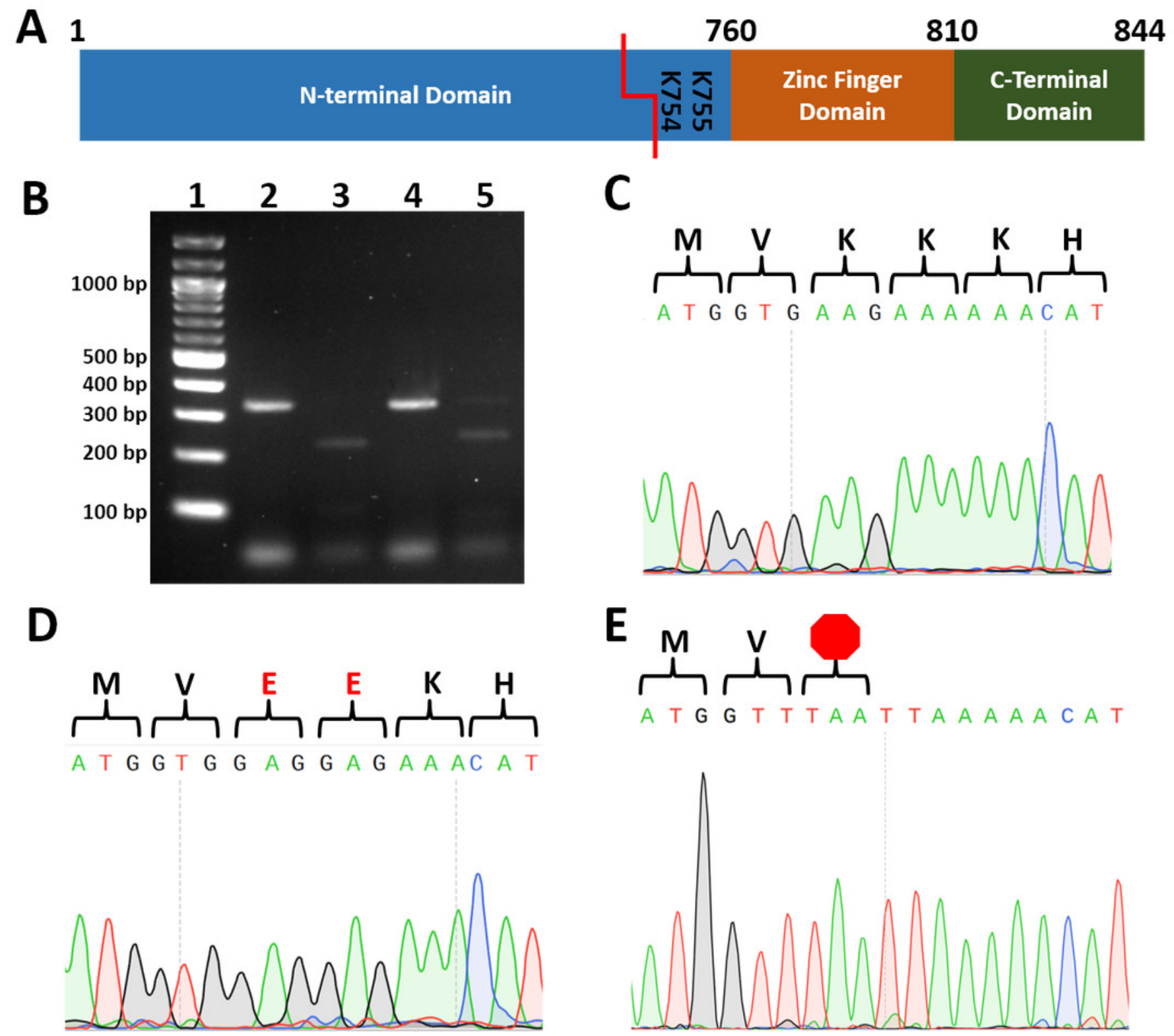




\section{Figure 2}

Assessment of UME6 mutations on filamentation and virulence.

A. Wild type, ume6-EE, and ume6-stopTAA C. albicans were cultured on Spider media at 30 and $37^{\circ} \mathrm{C}$ for 7 days and assessed for filamentation. B. Fourfold serial dilutions of wild type, ume6-EE, and ume6-stopTAA C. albicans were incubated on YPD media at $37{ }^{\circ} \mathrm{C}$ for 3 days.

C. Wild Type and ume6-stopTAA incubated in liquid Spider media were assessed for

filamentation after 24 hours. D. $10^{5}$ cfu of wild type, ume6-EE, and UME6-stopTAA C. albicans were injected into wax moth larvae, which were subsequently incubated at $37^{\circ} \mathrm{C}$. Survival of larvae was tracked over time. Wt was more virulent than ume6-EE $(P=0.036)$ and more virulent than ume6-stopTAA as well $(P<0.001)$. Additionally ume6-EE was more virulent than ume6-stopTAA $(P=0.003)$. Results represent total survival of 5 independent experiments with 10 larvae per treatment. Survival curves were created using the KaplanMeier method and statistical analyses were performed using the log rank test for multiple comparisons (IBM SPSS Statistics). 


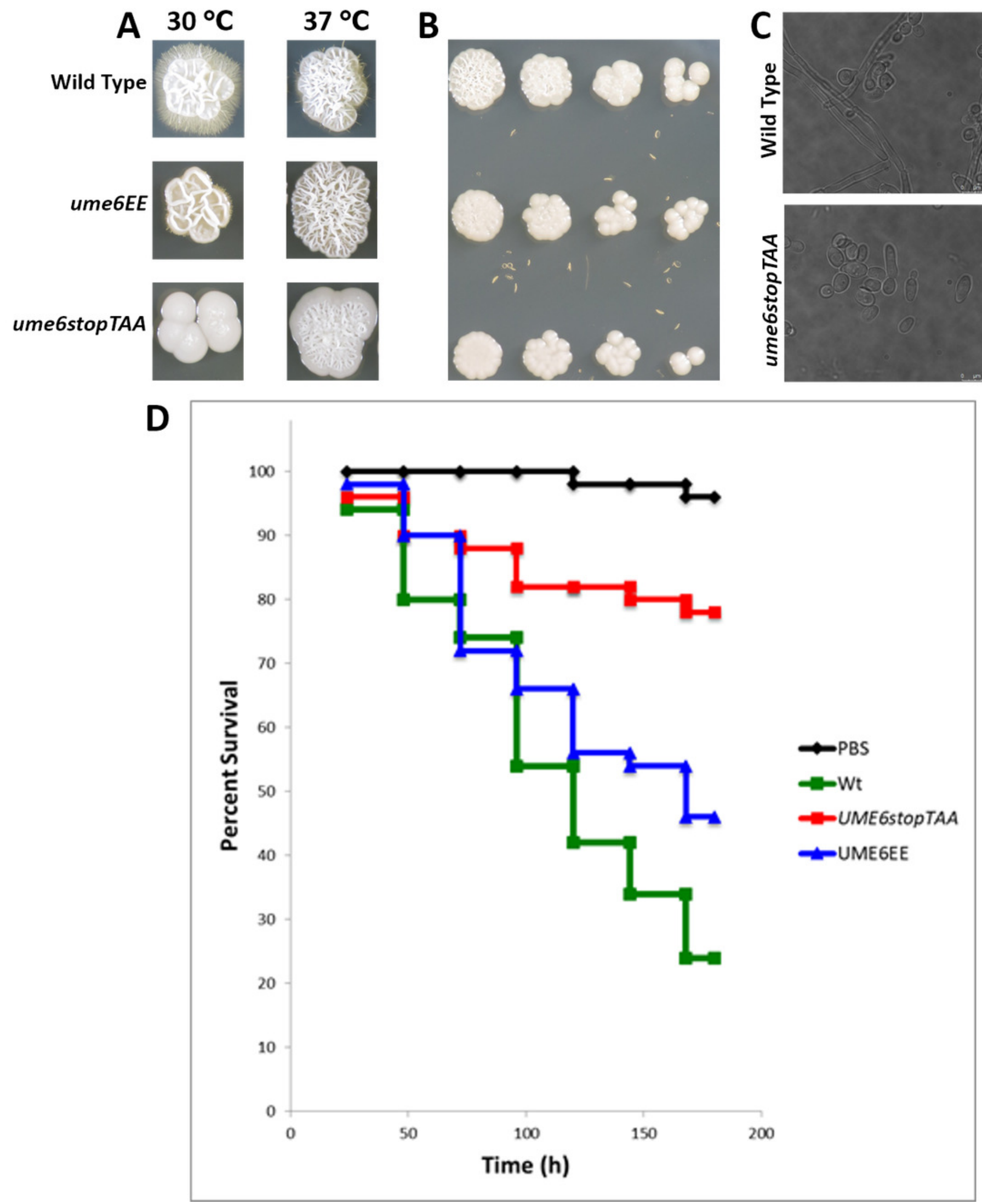




\section{Table $\mathbf{1}$ (on next page)}

Oligos used in this study.

Restriction Sites Sequences are bolded and capitalized in repair templates. Nucleotides added for cloning into the CRISPR plasmids are bolded and italicized in the guide primer sequences. 


\begin{tabular}{|l|l|l|}
\hline Oligo Name & Primer Sequence & Restriction Site \\
\hline Ume6 Guide 2 fr & ATTTGtacttctacttctaatccaaG & XXXXXX \\
\hline Ume6 Guide 2 rv & AAAACttggattagaagtagaagtaC & XXXXXX \\
\hline Ume6 ch primer 2 fr & ggtcatgatcatgatgatgaaaat & XXXXXX \\
\hline Ume6 ch primer 2 rv & ctccacaaattggtgtgacttc & XXXXXX \\
\hline Ume6 rp 2_Glu fr & atggcactaacaccaatactgattctacttctacttctaatccaatggtgGAGGAGaaac & BseRI \\
\hline Ume6 rp 2_Glu rv & catccttttttagatctaggtaataatcttcttcttgtatgtttCTCCTCcaccattgga & BseRI \\
\hline Ume6 rp 2_TAA fr & aatggcactaacaccaatactgattctacttctacttctaatccaatggtTTAATTAAaa & Pac1 \\
\hline Ume2 rp 2_TAA rv & tccttttttagatctaggtaataatcttcttcttgtatgtttTTAATTAAaccattggat & Pac1 \\
\hline Ume6 rp 2_TGA fr & atggcactaacaccaatactgattctacttctacttctaatccaatggtCTGAAGaaaca & Acu1 \\
\hline Ume6 rp 2_TGA rv & acatccttttttagatctaggtaataatcttcttcttgtatgtttCTTCAGaccattgga & Acu1 \\
\hline
\end{tabular}

1

2

3

4

5

6

7

8

9

10

11

12 


\section{Table 2 (on next page)}

Restriction sites that encode consecutive amino acids or stop codons.

Nucleotide code is as follows: $\mathbf{W}=\mathrm{A}$ or $\mathrm{T}, \mathbf{M}=\mathrm{A}$ or $\mathrm{C}, \mathbf{K}=\mathrm{G}$ or $\mathrm{T}, \mathbf{R}=\mathrm{A}$ or $\mathrm{G}, \mathbf{N}=\mathrm{A}$ T C or $\mathrm{G}$, I=cleavage site. Numbers in parentheses correspond to how far upstream a cut site will occur from the recognition sequence. 
1

\begin{tabular}{|c|c|l|}
\hline Translation Product & Codon & \multicolumn{1}{|c|}{ Restriction Enzyme Recognition Site Sequence } \\
\hline 2xAlanine & GCN & Bbvl GCAGCN (8/12), Fnu4HI GC/NGCN, Tsel G/CWGCN \\
\hline 2xArginine & CGN & Hpy99I CGWCGN/ \\
\hline 2xGlutamate & GAR & BseRI GAGGAG (10/8) \\
\hline 2xGlycine & GGC+GGA & Ecil GGCGGA (11/9) \\
\hline 1x Stop Codon & TAG & Spel A/CTAGT \\
\hline 1x Stop Codon & TAA & Pacl TTAAT/TAA \\
\hline 1x Stop Codon & TGA & Acul C TGAAG(16/14) \\
\hline 2x Stop Codon & TAG+TAA & I-Scel $\underline{\text { TAGGGATAACAGGGTAAT(-9/-13) }}$ \\
\hline
\end{tabular}

2 


\section{Table $\mathbf{3}$ (on next page)}

Strains used in this study. 


\begin{tabular}{|l|l|l|}
\hline Name & Genotype & $\begin{array}{l}\text { How strain is referenced in } \\
\text { text }\end{array}$ \\
\hline SC5314 & UME6:UME6 & Wild Type \\
\hline DAB898 & ume6-LYS754E LYS755E:ume6-LYS754E LYS755E & ume6-EE \\
\hline DAB894 & ume6-LYS754stop:ume6-LYS754stop & ume6-stopTAA \\
\hline
\end{tabular}

1 